\title{
Inter-collisional cutting of multi-walled carbon nanotubes by high-speed agitation
}

Ki Chul Park ${ }^{\mathrm{a}, *}$, Masatsugu Fujishige ${ }^{\mathrm{a}}$, Kenji Takeuchi ${ }^{\mathrm{a}}$, Susumu Arai ${ }^{\mathrm{b}}$, Shingo Morimoto ${ }^{\mathrm{c}}$, Morinobu Endo $^{\mathrm{a}, \mathrm{d}, *}$

${ }^{a}$ Department of Electrical and Electronic Engineering, Shinshu University, 4-17-1 Wakasato, Nagano 380-8553, Japan

${ }^{\mathrm{b}}$ Department of Chemistry and Material Engineering, Shinshu University, 4-17-1 Wakasato, Nagano 380-8553, Japan

${ }^{\mathrm{c}}$ Nagano Techno Foundation, 1-18-1 Wakasato, Nagano 380-0928, Japan

${ }^{\mathrm{d}}$ Institute of Carbon Science and Technology (ICST), Shinshu University, 4-17-1 Wakasato, Nagano 380-8553, Japan

*Corresponding authors. E-mail: endo@endomoribu.shinshu-u.ac.jp (M. Endo), kimuram@endomoribu.shinshu-u.ac.jp (K. C. Park) 


\begin{abstract}
High-speed agitation by a mixing blade has efficiently achieved the cutting of a large diameter (100-150 $\mathrm{nm}$ ) of multi-walled carbon nanotubes. The cutting process is caused by an inter-collision of the nanotubes with high transfer energy. The collision-induced cutting allows for the shortening of the nanotubes without serious damage of the sidewalls due to the cutting effect being limited to the collision points. Furthermore, the operation under ambient atmosphere introduces oxygen-containing functional groups to the cut nanotubes. The estimated length distribution has indicated that high-speed agitation achieves a large cutting effect during a short duration of several minutes.
\end{abstract}

Keywords: A. Nanostructures; C. Electron microscopy; D. Microstructure 


\section{Introduction}

One of the most promising applications of carbon nanotubes (CNTs) in technological fields is the utilization as fillers for advanced composite materials due to their unique chemical and physical properties [1-4]. In terms of the high mass productivity and purity of commercial products, multi-walled carbon nanotubes (MWCNTs) synthesized by catalytic chemical vapor deposition (CVD) are widely accepted to be suitable for reinforcing fillers of polymer composites. The effective utilization of CNTs as reinforcing fillers requires their homogeneous dispersion throughout the polymer matrices without degenerating the integrity of nanotubes. However, the CVD-derived MWCNTs are commonly in an entangled agglomerate state because of high aspect ratios $(>100)$ and structural defects of bends and kinks. Therefore, disentanglement of agglomerates without damaging the nanotube structure is indispensable for improving the mechanical properties of polymer composites. Furthermore, the mechanical strength of CNT-polymer composites is enhanced by an interfacial adhesion, allowing for load transfer from polymer matrices to nanotubes. This emphasizes that chemical and structural modification of nanotube surface, dependent on the selection of matrix polymers, is required to enhance the final load transfer effect [5].

As the simplest approach, the entanglement of CNTs can be solved by chemical and mechanical cutting techniques. Thus far, a variety of cutting methods have been reported, including acid [6,7] and thermal [8,9] oxidation, ball milling [10-20], ultrasonication [21], and ultrasound-assisted cutting in mixed acids [22,23] and in a polymer-dissolved organic solvent [24]. Dry ball milling [10-12] and diamond-grain grinding [25,26], which are analogous in principle, have effectively cut CNTs to solve the nanotube entanglement. However, the high-impact process causes crucial damage of the nanotube structure [10-12]. In contrast, wet [11], low-impact [17], and polymer-mediated [18-20] ball-milling processes and cyclodextrin-mediated grinding [27] have achieved less destructive cutting with almost no structural collapse. Disadvantageously, the low-impact or energy-dispersive processes require much time or cycled operations to reach desired and homogeneous average length distribution. The oxidative cutting methods [6-8] have shortened CNTs with the opening of nanotube closed ends and functionalization of the opened edges and sidewalls. The resulting CNTs can be favorably dispersed in polar solvents due to the effect of short lengths and the hydrophilic functional groups. Similar effects on nanotube dispersion are expected for polymer matrices with polar groups. Note that the reaction in the chemical cutting process is initiated on the more reactive kink and defect sites on the nanotube ends and sidewalls. The selectivity for reaction sites means that oxidative cutting would be largely dependent on the structure of the nanotubes employed. Furthermore, prolonged oxidation to reach the desired length distribution causes serious weight loss of CNTs because of erosion and thinning of the shortened CNTs from the more reactive edge sites. Cutting and opening of CNTs by ultrasonication also show the similar selectivity for reaction sites. In the prolonged operation, attention should be paid for the induction of the defects such as wall fracture, buckling and dislocation onto the nanotube sidewalls [28].

Here we report a unique cutting method of MWCNTs with a large diameter of 100-150 nm by highspeed rotating of a mixing blade. The cutting is promoted by the inter-collision of CNTs with high transfer energy. The high-impact process requires short operation time of only several minutes for cutting CNTs. More importantly, the cutting effect is limited mainly to the collision points. Therefore, cutting of CNTs can be achieved without collapse of the tubular structures and any deformation of the initial graphitic structures, different from other high-impact mechanical and chemical processes. Furthermore, cutting process under ambient atmosphere is accompanied by the introduction of oxygencontaining functional groups to the cut nanotubes.

\section{Experimental}

The CVD-derived MWCNTs were purchased from Showa Denko Co. (diameter: 100-150 nm and length: $10-20 \mu \mathrm{m})$. The MWCNTs were supplied as purified products, that is, the metal catalysts used in the CVD process were removed by heat treatment at $2800^{\circ} \mathrm{C}$ under inert atmosphere. In addition, the heat treatment makes the as-prepared MWCNTs more graphitized. Thus, the as-received MWCNTs were used without further purification. Experiments for the cutting of MWCNTs were conducted using a mill mixer (Wonder Blender WB-1, Osaka Chemical Co.). The mill mixer is assembled by a cylinder- 
type vessel (inner volume: $150 \mathrm{~mL}$ ), a lid, and a mixing blade equipped in the center of the vessel bottom. The agitation of the CNTs by the mixing blade was conducted at 25,000 rpm for 1 to $15 \mathrm{~min}$ in dry conditions under ambient atmosphere.

The structural and morphological investigation was performed by transmission electron microscopy (TEM), X-ray diffraction (XRD), Raman spectroscopy, and scanning electron microscopy (SEM). The TEM images were recorded on a JEOL JEM-2100F instrument operated at an acceleration voltage of $120 \mathrm{kV}$. The XRD patterns were recorded on a Rigaku RINT 2200V/PC-SV diffractometer with a Cu $\mathrm{K} \alpha$ source operated at $40 \mathrm{kV}$ and $20 \mathrm{~mA}$. The Raman spectra were recorded on Kaiser Optical Systems HoloLab-5000 apparatus (incident excitation: $532 \mathrm{~nm}$ of Nd:YAG laser). The SEM images were recorded on a JEOL JSM-7000F/IV instrument operated at an acceleration voltage of $15 \mathrm{kV}$. The highresolution SEM was measured on a Hitachi S-5500 instrument operated at an acceleration voltage of 30 $\mathrm{kV}$. The length distribution of the cut nanotubes was determined by a Shimadzu SALD-7000 laserdiffraction particle size analyzer (incident laser wavelength: $405 \mathrm{~nm}$, power: $3 \mathrm{~mW}$ ). The surface chemistry of the cut CNTs was investigated by X-ray photoelectron spectroscopy (XPS) performed on a JEOL JPS-9200 apparatus. The entire XP spectra were referenced to the C1s peak of the graphite (284.6 $\mathrm{eV})$.

\section{Results and discussion}

Fig. 1 shows the SEM images of the original and the MWCNTs shortened by the mill mixer for 1 to 15 min. Clearly, the lengths of the CNTs treated for 10 min (Fig. 1(d), (e)) are shorter than those of the original nanotubes (Fig. 1(a), (b)). The cutting for 10 min caused a decrease of ca. 37\% in sample volume, which was no longer changed by elongating the operation time. The SEM images also show no remarkable difference in length between the samples for $10 \mathrm{~min}$ (Fig. 1(d)) and for 15 min (Fig. 1(f)). If anything, the prolonged operation needlessly produced particulate impurities. Note that particulate impurities were observed in the sample operated for $1 \mathrm{~min}$, although the amount was much smaller compared to the longer-operated samples. In a high-impact ball-milling process, the prolonged time of grinding causes collapse of graphitic tubular structure of CNTs into granules and sheets of amorphous carbon phase [10-12]. In contrast, high-speed agitation produces particulate impurities even during quite short durations. This suggests that the two different processes would contribute to the cutting of CNTs and the production of particulate impurities.

The basic principle of functioning of high-speed mill mixer lies in pulverization of the samples by trituration, cataclasis, inter-collision of sample molecules with high transfer energy, and their collision with the rotating blade or the vessel wall. The contribution of trituration and cataclasis to nanotube cutting would not be significant due to the small size of the nanotubes. Primarily, a high-energy transfer of CNTs caused by high-speed agitation would relate to the cutting mechanism. It is undoubted that the frequency factor of inter-collision of nanotubes is quite large due to the extremely fast transfer and high population. Therefore, the observed cutting effect would be attributed to the high-energy inter-collision of nanotubes. This implies that the high impact that is localized in the collision part of nanotubes does not affect the non-collision part of graphitic layer structures (see below for details). In contrast to the localized impact, collisions with the blade or vessel walls are considered to highly impact the expanded area of nanotubes. Therefore, particulate impurities would be produced by collision of nanotubes with the blade or vessel walls.

The magnified SEM and TEM images of the cut ends of the shortened nanotubes are shown in Fig. 2. All of the cut ends observed are opened and jagged. Furthermore, the cut edges of graphitic layers are not aligned but have some gaps. The appearance of cut ends is different from the well-aligned end shape of the MWCNTs cut by the mechanical stress onto the bending and kinking defect sites [15]. Therefore, the disordered cut ends appear to support that cutting can be achieved on random collision points independent of the structural defects of nanotubes. As described above, prolonged operation almost no longer shows further shortening of the nanotubes. This might be attributed to the reduction of molecular weight in the cut nanotubes, because kinetic and collision energies are largely dependent on the molecular weight. Fig. 3 shows the sidewalls and intact tips of cut MWCNTs. The absence of definite 
damages on the graphitic layers and the original closed end support that high impact is localized to the collision part.

Figs. 4 and 5 show the XRD and Raman spectra of the original and the cut MWCNTs. The XRD spectra showed similar diffraction angle and the full-width at half-maximum (FWHM) for graphite (002) peaks. The Raman spectra also showed no variation of position and FWHM of the graphite E2 $g_{2}$ $\mathrm{G}$ peaks. The spectroscopic results imply no serious deformation of the original graphitic structures after cutting. This supports that the cutting is achieved by localized high impact. In the Raman spectra, the relative intensity $\mathrm{I}_{\mathrm{D}} / \mathrm{I}_{\mathrm{G}}$ of the $\mathrm{D}$ to the $\mathrm{G}$ peak increases with operation time. The $\mathrm{D}$ peak originates from the symmetrical breathing vibration of the aromatic rings located in the edge sites and defects of the graphite network. As described above, the TEM images of the sidewalls and the spectroscopic information about the graphitic structures show no definite damage. Therefore, the increase of the $I_{D} / I_{G}$ ratio would be attributed mainly to the increase of the edge sites with progressive of cutting. At the long operation time of $10 \mathrm{~min}$, the intensity of the D' peak increases parallel to that of the D peak. Similar to D peaks, D' peaks are developed by a crystalline effect. However, note that D' peaks, which arise from the same vibrational motion as $G$ peaks, are closely related to carbon atoms existing on different environments of the external surface of the crystals [29]. Therefore, the remarkable development of the D' peak seems to suggest a possibility that the long-time operation might introduce the structural defects into the external surface by insufficient impacts for cutting. Even if that is the case, the results of microscopic and spectroscopic analyses clearly indicate structural integrity of most of the original graphitic layers.

Fig. 6 shows the XPS C1s peaks of the original and the cut MWCNTs. The composition ratios of carbon and oxygen calculated from the $\mathrm{C} 1 \mathrm{~s}$ and $\mathrm{O} 1 \mathrm{~s}$ (not shown here) peaks are listed in Table 1. The composition ratios show the obvious increase of oxygen proportion at the long operation time of $10 \mathrm{~min}$. The curve fitting of the $\mathrm{C} 1 \mathrm{~s}$ peaks exhibited three different environments of carbon. The main components of the peak can be assigned to $\mathrm{sp}^{2} / \mathrm{sp}^{3}$-carbon, $\mathrm{C}-\mathrm{O}$, and $\mathrm{C}=\mathrm{O}[30,31]$. The relative intensity of $\mathrm{C}=\mathrm{O}$ has increased with operation time. It is therefore concluded that collision-induced cutting under ambient atmosphere introduces oxygen-containing functional groups to the cut nanotubes. The structural integrity of graphitic layers of the cut nanotubes implies that the functional groups would be introduced mainly into the edge sites of the cut ends. However, the oxygen proportion of the sample operated for $10 \mathrm{~min}$ is comparable to that of the acid-oxidized sample (acid treatment: reflux in the mixed acid of $60 \%$-nitric acid $/ 90 \%$-sulfuric acid $(\mathrm{v} / \mathrm{v}=3 / 2)$ at $120^{\circ} \mathrm{C}$ for $\left.12 \mathrm{~h}\right)$. Considering the results of the Raman spectra, therefore, the long-time agitation might introduce functional groups to the possible structural defects of the external surface.

The length distribution of cut nanotubes has been commonly estimated from atomic force microscopy, SEM, and TEM images. However, the clumps of the cut CNTs hinder proper estimation of length distribution by microscopic methods. Furthermore, hundreds of images must be analyzed for statistical validity. In this work, we tried to estimate the length distribution of the cut nanotubes by a particledistribution analyzer employing laser-diffraction method. The particle size distribution (PSD) estimated by the laser-diffraction analyzer is calculated according to Mie theory, which deals with diffraction and scattering of light on spherical particles. The MWCNTs used in our work have diameters of 100-150 nm, lengths of 10-20 $\mu \mathrm{m}$, and the incident irradiation of the particle size analyzer is $\lambda=405 \mathrm{~nm}$. Therefore, the Mie parameters, $\alpha(=\pi \mathrm{d} / \lambda, d$ particle diameter, $\lambda=$ wavelength of incident light [32]), for categorizing the scattering behavior by the interaction between a single particle and incident light range from 0.78 to 1.16 for nanotube breadths and from 77 to 155 for lengths. The $\alpha$ values lie in the region of Mie scattering and geometric optic scattering [32]. Supposing that the samples are optically isotropic spheres, therefore, the particle size can be determined from the scattered light distribution.

In the apparatus employed, the suspension of the CNTs flows through the optical cell of the narrow space between the two parallel glass walls (optical path length: $6 \mathrm{~mm}$ ). Therefore, it is supposed that the tubular particles have a tendency to orient along the flow direction. The incident light applied vertically to the glass cell would be scattered by the oriented nanotubes to provide dissymmetrical scattered light pattern depending on the breadth and length of the nanotubes. Therefore, in the case of well-dispersed long tubular particles, the smaller breadth and the larger length could be individually recognized to 
provide the mixed distribution profiles. In fact, a previous work has reported the influence of particle shape on the PSD estimated by the laser-diffraction and scattering method [33]. The report described that the average $10 \%$ and $90 \%$ diameters measured for rod-like particles were, respectively, smaller and larger than the average diameter determined by the electrical sensing zone method (which provides the particle diameter independent of the anisotropy of particle shape). Therefore, the authors have concluded that the average $10 \%$ and $90 \%$ diameters provide information on the breadth and length of rod-like particles. Fig. 7 shows the PSD of the original and the cut MWCNTs. The observed peak in the large-size region is in good consistency with the quoted length $(10-20 \mu \mathrm{m})$ of the MWCNTs (the peak maximum: ca. $12 \mu \mathrm{m}$ ). Furthermore, the peak is shifted to the smaller side with operation time. In contrast, the peak in the small-size region is constant independent of the operation time, which is in good consistency with the quoted diameter (100-150 nm) of the MWCNTs. It is therefore concluded that the laser-diffraction particle size analyzer provides an adequate distribution for the breadth and length of MWCNTs. In the sample operated for $1 \mathrm{~min}$, the length of the cut nanotubes is in the range of ca. 1 to $15 \mu \mathrm{m}$ (the peak maximum: ca. $4.2 \mu \mathrm{m}$ ). Prolonged operation up to $10 \mathrm{~min}$ further shortened the nanotube length to the range of ca. 0.6 to $10 \mu \mathrm{m}$ (the peak maximum: ca. $1.8 \mu \mathrm{m}$ ). In comparison among the peak maxima, short-time operations for 1 and 10 min provide large cutting effects, i.e., 35\% and $15 \%$ lengths of the original MWCNTs. At the same time, the prolonged cutting process increases particulate impurities given as shoulder peaks in the range from 0.2-1 $\mu \mathrm{m}$. However, the proportion of particulate impurities can be kept lower by short duration. Finally, it should be noted that the particle size analyzer is not applicable to much smaller CNTs than employed in our work. Small particles with Mie parameter $\alpha<<1$ show Rayleigh scattering, which provide symmetry of scattered light not to be used for the determination of PSD.

\section{Conclusion}

MWCNTs (diameter: 100-150 nm, length: $10-20 \mu \mathrm{m}$ ) have been effectively cut for 1 to 10 min by high-speed agitation, where inter-collision of nanotubes with high transfer energy contributes to the cutting. The inter-collisional cutting is accompanied by the production of paticulate impurities, probably, because of collision between nanotubes and the blade and vessel wall. However, the proportion of the particulate impurities is not so high for the short duration. More importantly, inter-collisional cutting allows for shortening of nanotubes without crucial damages of the original graphitic layers, because the high impact is limited to the collision points. Furthermore, the cutting process under ambient atmosphere introduces oxygen-containing functional groups into the cut nanotubes. Our results emphasize that the diameter and length distribution of the cut MWCNTs can be estimated by the laserdiffraction particle size analyzer for nanotubes with the size categorized into Mie scattering. The estimated length distribution indicates that cutting by high-speed agitation achieves large cutting efficiency for short duration of several minutes.

\section{Acknowledgement}

The authors gratefully acknowledge the Ministry of Education, Culture, Sports, Science, and Technology of Japan for the financial support of the second stage Intellectual Cluster Project.

\section{References}

[1] E.W. Wong, P.E. Sheehan, C.M. Lieber, Science 277 (1997) 1971-1975.

[2] J.-P. Salvetat, G.A.D. Briggs, J.-M. Bonard, R.R. Bacsa, A.J. Kulik, T. Stöckli, L. Forró, Phys. Rev. Lett. 82 (1999) 944-947.

[3] S. Frank, P. Poncharal, Z.L. Wang, W.A. de Heer, Science 280 (1998) 1744-1746.

[4] P. Kim, L. Shi, A. Majumdar, P.L. McEuen, Phys. Rev. Lett. 87 (2001) 215502/1-215502/4. 
[5] R. Andrews, D. Jacques, D. Qian, T. Rantell, Acc. Chem. Res. 35 (2002) 1008-1017.

[6] J. Li, Y. Zhang, Appl. Surf. Sci. 252 (2006) 2944-2948.

[7] J. Liu, A.G. Rinzler, H. Dai, J.H. Hafner, R.K. Bradley, P.J. Boul, A. Lu, T. Iverson, K. Shelimov, C.B. Huffman, F. Rodriguez-Macias, Y.-S. Shon, T.R. Lee, D.T. Colbert, R.E. Smalley, Science 280 (1998) 1253-1256.

[8] M.Q. Tran, C. Tridech, A. Alfrey, A. Bismarck, M.S.P. Shaffer, Carbon 45 (2007) 2341-2350.

[9] Z. Kang, E. Wang, B. Mao, Z. Su, C. Tian, L. Xu, Mater. Lett. 60 (2006) 2266-2269.

[10] Z. Zhang, Z. Sun, Y. Chen, Appl. Surf. Sci. 253 (2007) 3292-3297.

[11] J.-H. Ahn, H.-S. Shin, Y.-J. Kim, H. Chung, J. Alloys Compd. 434-435 (2007) 428-432.

[12] Y.B. Li, B.Q. Wei, J. Liang, Q. Yu, D.H. Wu, Carbon 37 (1999) 493-497.

[13] Y.F. Sun, A.M. Zhang, Y. Yin, Y.M. Dong, Y.C. Cui, X. Zhang, J.M. Hong, Mater. Chem. Phys. 101 (2007) 30-34.

[14] C. Zapata-Massot, N.L. Bolay, Chem. Eng. Proc. (2007), doi:10.1016/j.cep.2007.05.001.

[15] Z. Kónya, J. Zhu, K. Niesz, D. Mehn, I. Kiricsi, Carbon 42 (2004) 2001-2008.

[16] K. Niesz, A. Siska, I. Vesselényi, K. Hernadi, D. Méhn, G. Galbács, Z. Kónya, I. Kiricsi, Catal. Today 76 (2002) 3-10.

[17] Á. Kukovecz, T. Kanyó, Z. Kónya, I. Kiricsi, Carbon 43 (2005) 994-1000.

[18] W. Shao, Q. Wang, F. Wang, Y. Chen, Carbon 44 (2006) 2708-2714.

[19] L. Chen, X.-J. Pang, Q.-T. Zhang, Z.-L. Yu, Mater. Lett. 60 (2006) 241-244.

[20] L. Chen, M.-Z. Qu, G.-M. Zhou, B.-L. Zhang, Z.-L. Yu, Mater. Lett. 58 (2004) 3737-3740.

[21] K.B. Shelimov, R.O. Esenaliev, A.G. Rinzler, C.B. Huffman, R.E. Smalley, Chem. Phys. Lett. 282 (1998) 429-434.

[22] T. Saito, K. Matsushige, K. Tanaka, Physica B 323 (2002) 280-283.

[23] E. Farkas, M.E. Anderson, Z. Chen, A.G. Rinzler, Chem. Phys. Lett. 363 (2002) 111-116.

[24] M. Yudasaka, M. Zhang, C. Jabs, S. Iijima, Appl. Phys. A Mater. Sci. Proc. 71 (2000) 449-451.

[25] I. Stepanek, G. Maurin, P. Bernier, J. Gavillet, A. Loiseau, R. Edwards, O. Jaschinski, Chem. Phys. Lett. 331 (2000) 125-131.

[26] G. Maurin, I. Stepanek, P. Bernier, J.-F. Colomer, J. B. Nagy, F. Henn, Carbon 39 (2001) 1273 1278.

[27] J. Chen, M.J. Dyer, M.-F. Yu, J. Am. Chem. Soc. 123 (2001) 6201-6202.

[28] K.L. Lu, R.M. Rago, Y.K. Chen, M.L.H. Green, P.J.F. Harris, S.C. Tsang, Carbon 34 (1996) 814816.

[29] R. Escribano, J.J. Sloan, N. Siddique, N. Sze, T. Dudev, Vib. Spectrosc. 26 (2001) 179-186. 
[30] R. Yu, L. Chen, Q. Liu, J. Lin, K.-L. Tan, S.C. Ng, H.S.O. Chan, G.-Q. Xu, T.S.A. Hor, Chem. Mater. 10 (1998) 718-722.

[31] A. Jitianu, T. Cacciaguerra, R. Benoit, S. Delpeux, F. Béguin, S. Bonnamy, Carbon 42 (2004) 1147-1151.

[32] U. Teipel, Chem. Eng. Technol. 25 (2002) 13-21.

[33] M. Naito, O. Hayakawa, K. Nakahira, H. Mori, J. Tsubaki, Powder Technol. 100 (1998) 52-60. 


\section{Figure captions}

Fig. 1. SEM images of the cut MWCNTs: (a), (b) the original MWCNTs. The MWCNTs were cut by high-speed agitation for (c) $1 \mathrm{~min}$, (d) and (e) $10 \mathrm{~min}$, and (f) $15 \mathrm{~min}$.

Fig. 2. (a) SEM and (b), (c) TEM images of the cut ends. The MWCNTs were cut for (a) and (c) 10, and (b) $1 \mathrm{~min}$.

Fig. 3. TEM images of the intact sidewalls and the closed ends. The MWCNTs were cut for (a) and (d) $10 \mathrm{~min}$, and (b) and (c) $1 \mathrm{~min}$.

Fig. 4. XRD patterns of (a) the original and the MWCNTs cut for (b) 1 and (c) $10 \mathrm{~min}$.

Fig. 5. Raman spectra of (a) the original and the MWCNTs cut for (b) 1 and (c) 10 min.

Fig. 6. XPS C1s peaks of (a) the original and the MWCNTs cut for (b) 1 and (c) $10 \mathrm{~min}$.

Fig. 7. Length distribution of the cut MWCNTs. (a) Original MWCNTs. The MWCNTs were cut for (b) 1 and (c) $10 \mathrm{~min}$.

\section{Table captions}

\section{Table 1}

Carbon and oxygen proportions of the cut MWCNTs calculated from C1s and O1s XPS spectra Acid-oxidized samples were prepared by refluxing the original MWCNTs in the mixed acid $(60 \%$ nitric acid $/ 90 \%$-sulfuric acid, $v / \mathrm{v}=3 / 2$ ) at $120^{\circ} \mathrm{C}$ for $12 \mathrm{~h}$. 
Table 1

\begin{tabular}{ccc}
\hline Sample & C (at\%) & O (at\%) \\
\hline Original MWCNTs & 97.9 & 2.1 \\
MWCNTs cut for 1 min & 98.3 & 1.7 \\
MWCNTs cut for 10 min & 95.8 & 4.2 \\
Acid-oxidized MWCNTs & 95.7 & 4.3 \\
\hline
\end{tabular}


Fig. 1
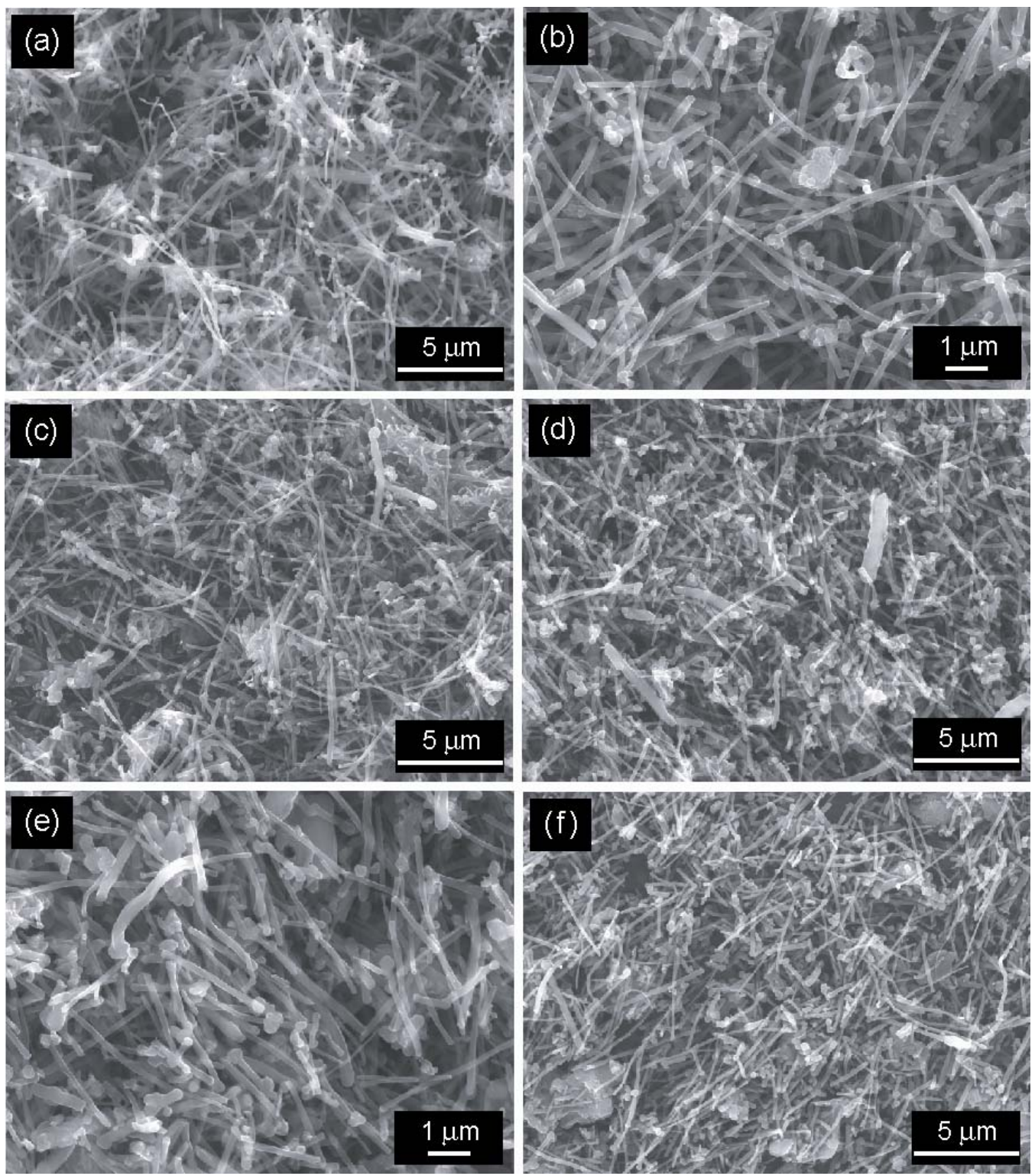
Fig. 2
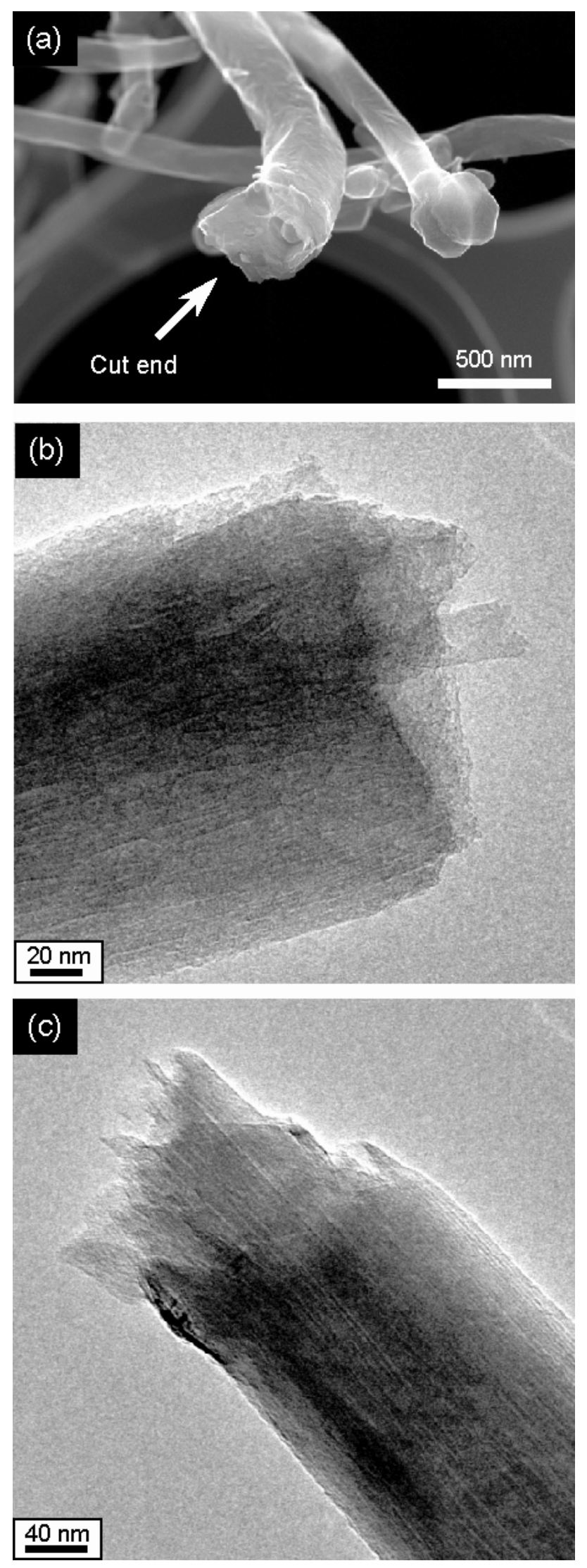
Fig. 3
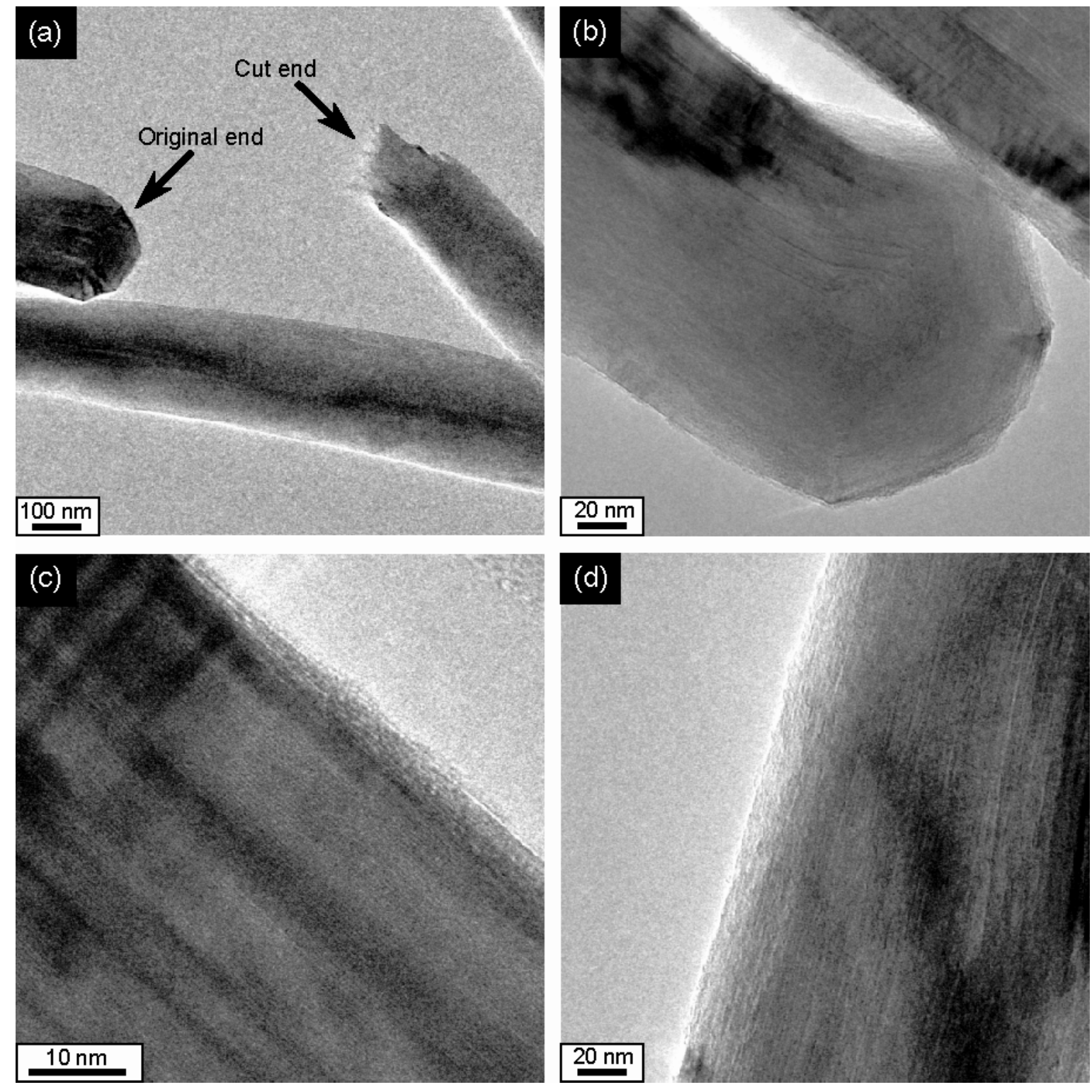
Fig. 4

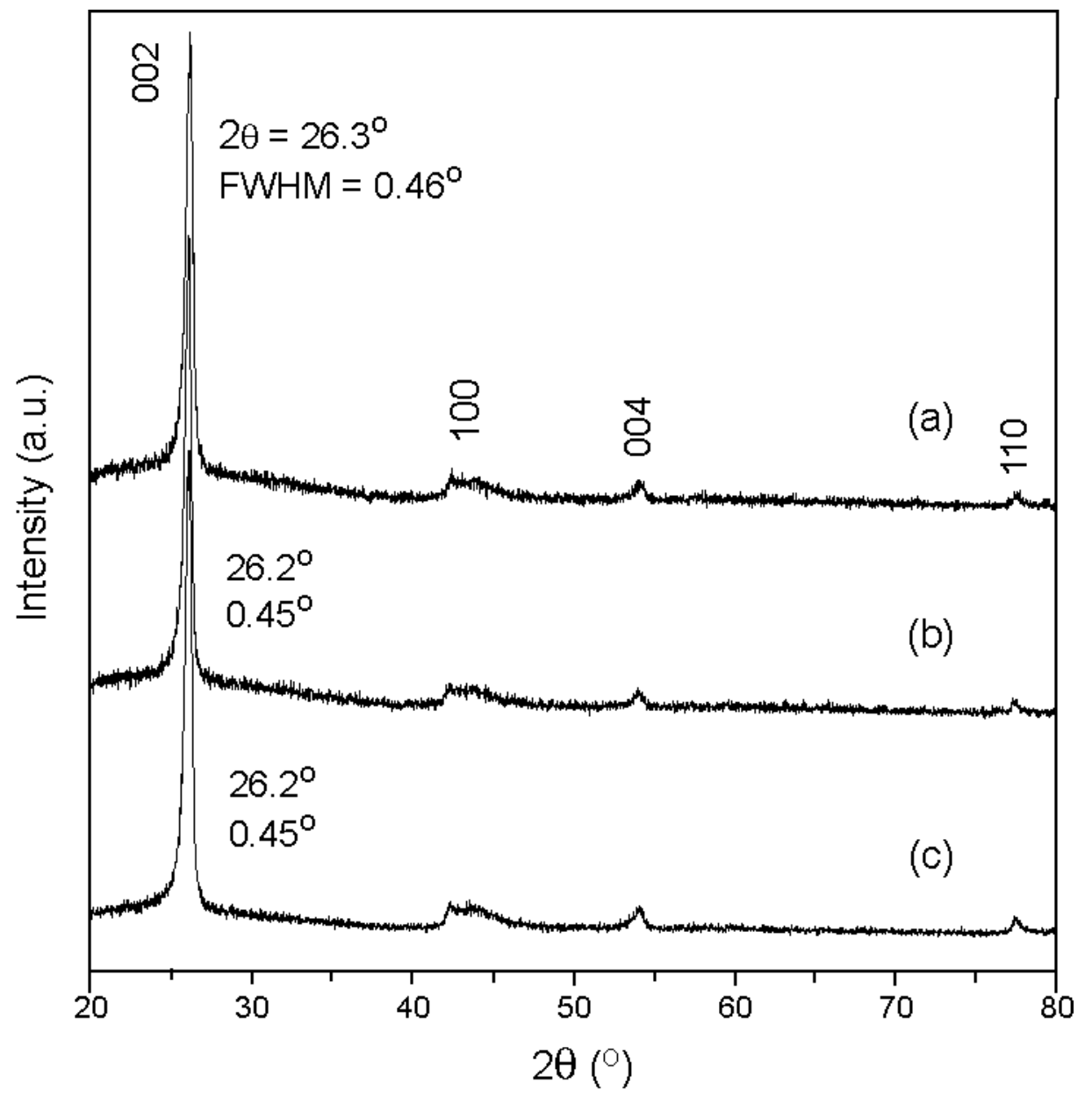


Fig. 5

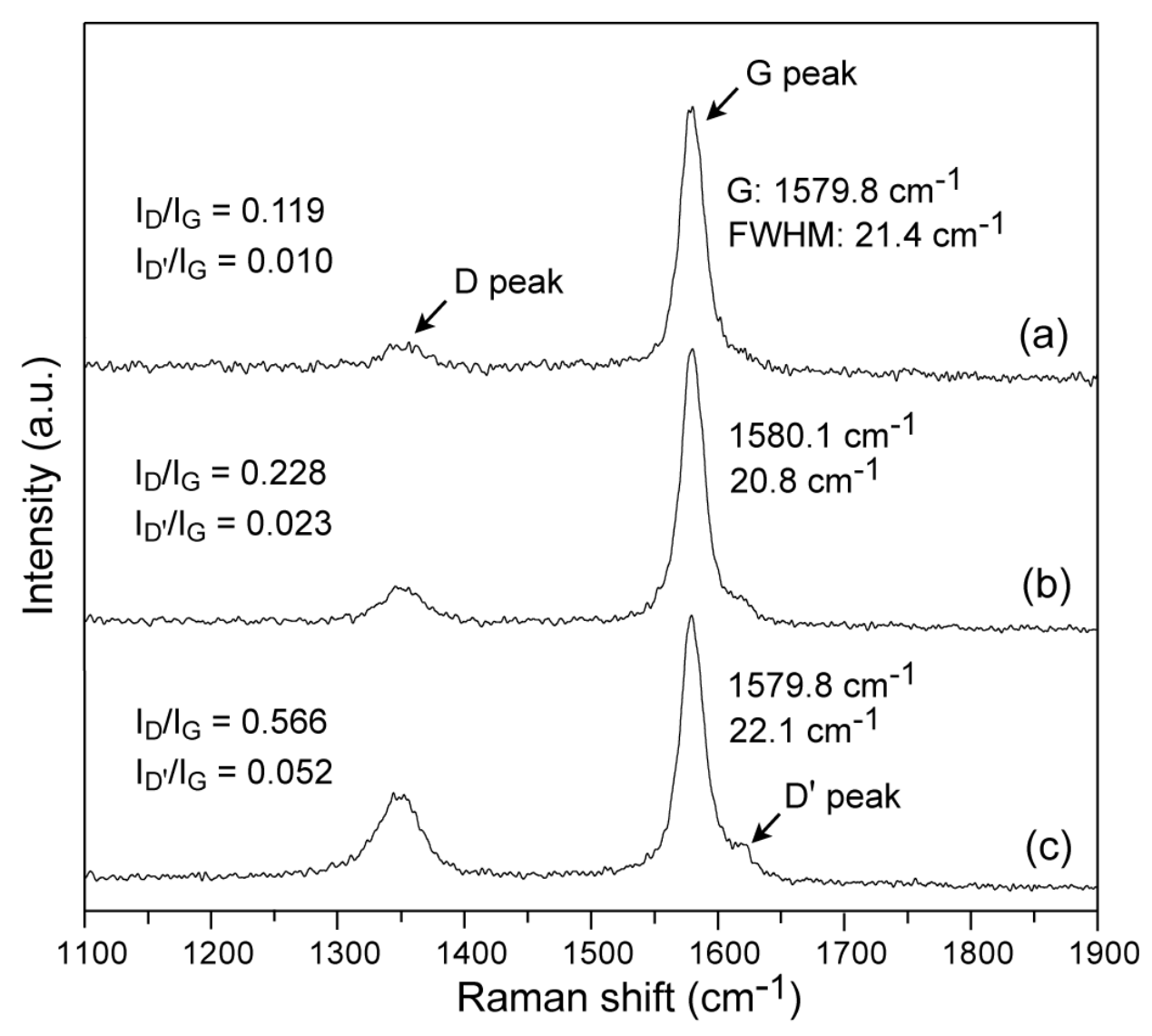


Fig. 6

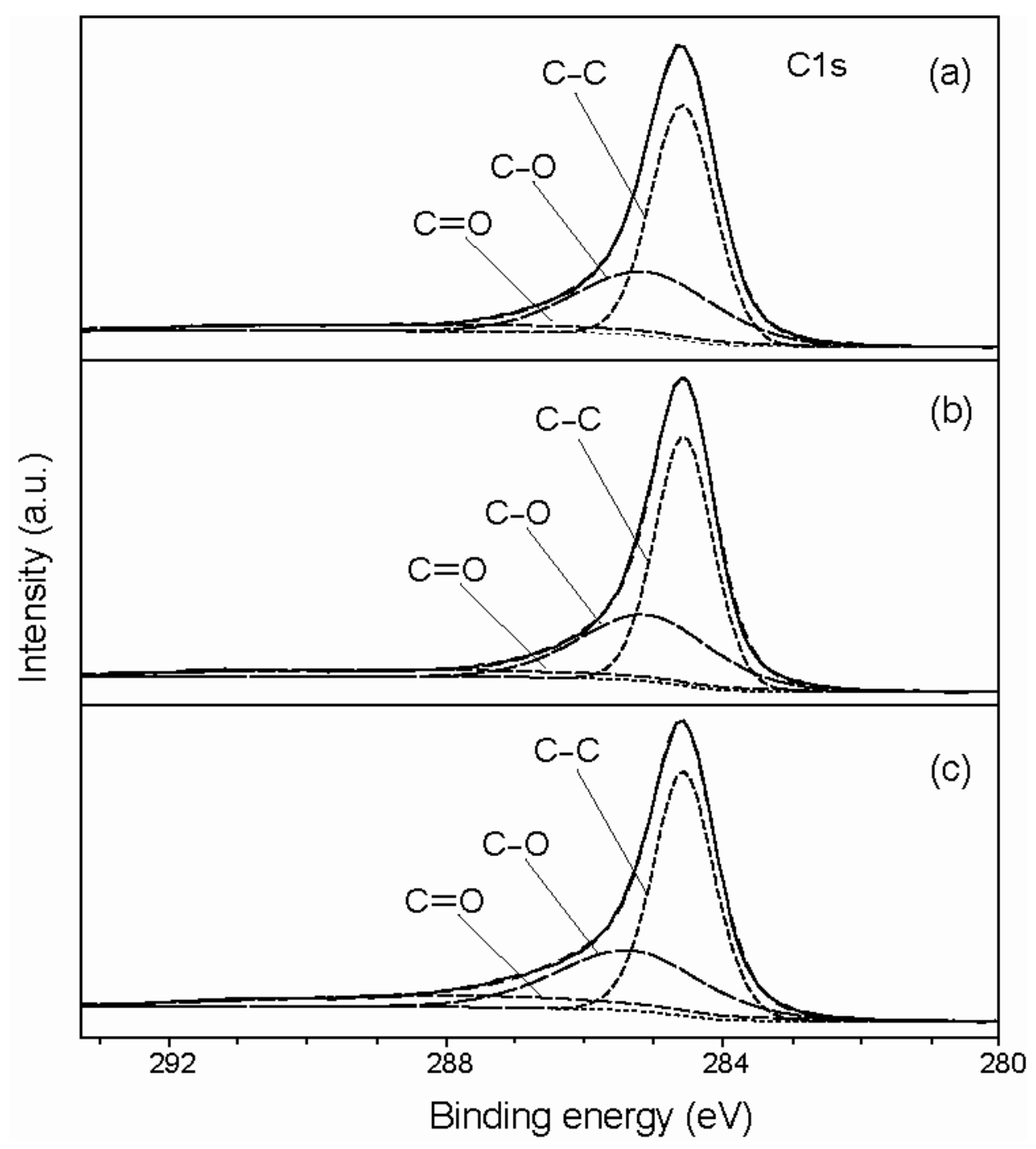


Fig. 7

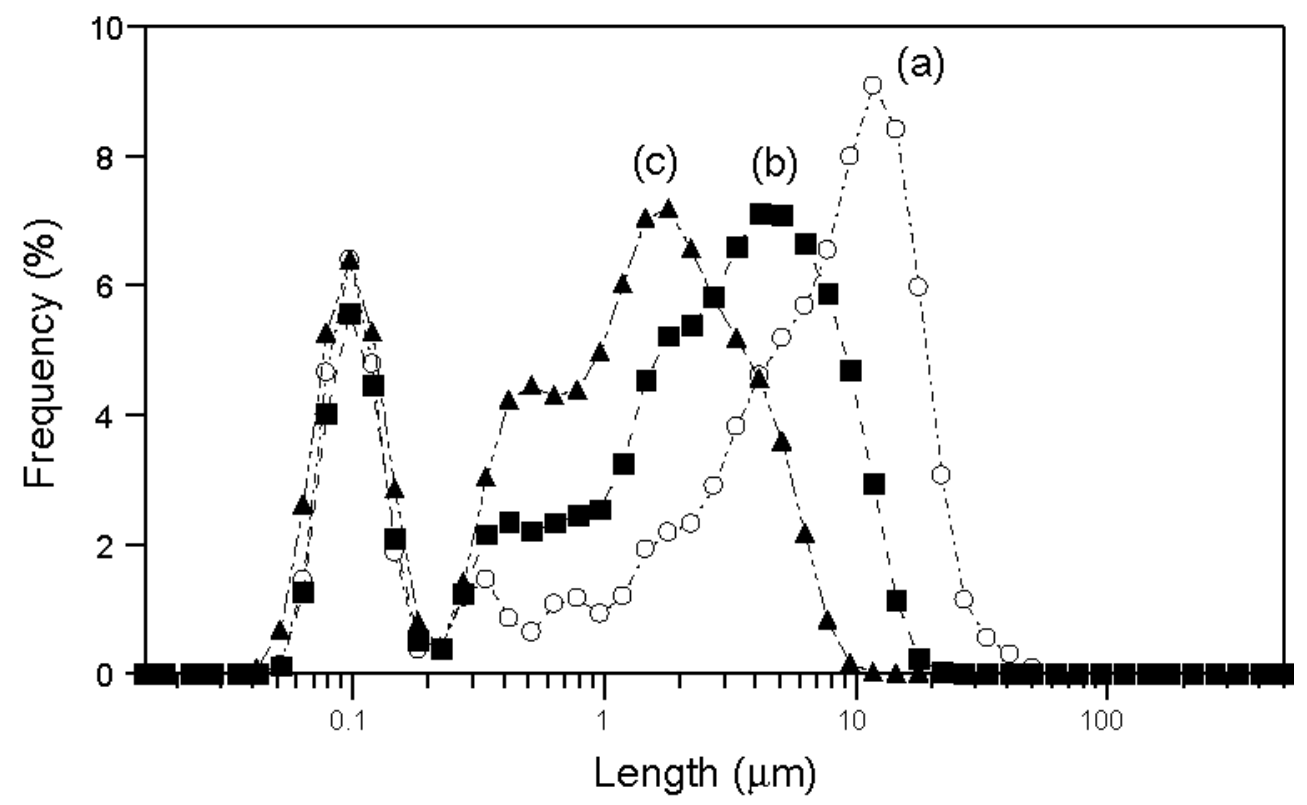

